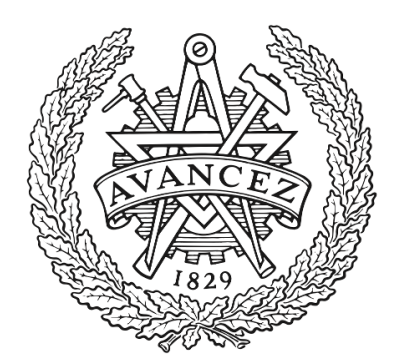

CHALMERS

UNIVERSITY OF TECHNOLOGY

\title{
Design and Fabrication of a High-Gain 60-GHz Corrugated Slot Antenna Array With Ridge Gap Waveguide Distribution Layer
}

Downloaded from: https://research.chalmers.se, 2023-04-26 14:20 UTC

Citation for the original published paper (version of record):

Zarifi, D., Farahbakhsh, A., Uz Zaman, A. et al (2016). Design and Fabrication of a High-Gain 60-GHz Corrugated Slot Antenna Array With Ridge Gap

Waveguide Distribution Layer. IEEE Transactions on Antennas and Propagation, 64(7): 2905-2913. http://dx.doi.org/10.1109/tap.2016.2565682

N.B. When citing this work, cite the original published paper.

C2016 IEEE. Personal use of this material is permitted.

However, permission to reprint/republish this material for advertising or promotional purposes 


\title{
Design and Fabrication of A High-Gain $60 \mathrm{GHz}$ Corrugated Slot Antenna Array with Ridge Gap Waveguide Distribution Layer
}

\author{
DavoudZarifi, Ali Farahbakhsh, Ashraf Uz Zaman, Per-Simon Kildal, Fellow, IEEE
}

\begin{abstract}
A wideband, high gain high efficiency $16 \times 16$-element slot antenna array is presented for $60 \mathrm{GHz}$ band applications. The antenna is designed based on gap waveguide technology. The most important advantage of using this technology is its ability to decrease complexity and cost of fabrication because there is no requirement of electrical contact between the three layers of the antenna structure. The three layers are a corrugated metal plate with radiating slots, a sub-array cavity layer, and a ridge gap waveguide feed network layer. The corporate feed network is realized by a texture of pins and guiding ridges in a metal plate. Also, in order to excite the antenna with a standard V-band rectangular waveguide, a transition from ridge gap waveguide to WR-15 is designed. The radiating elements, corrugations, cavity layer, power dividers and transition are designed and optimized to suppress the reflection coefficient at the input WR-15 port over the desired frequency range from 57 to $66 \mathrm{GHz}$. Finally, the 16×16-element slot antenna array is fabricated by standard milling technology. Measured results demonstrate about $16 \%$ of reflection coefficient bandwidth $\left(\left|S_{11}\right|<-10 \mathrm{~dB}\right)$ covering 56-65.7 $\mathbf{G H z}$ frequency range, and the measured gain is larger than 32.5 dBi over the band with more than $70 \%$ antenna efficiency.
\end{abstract}

Index Terms - Slot array antenna, corporate feed network, gap waveguide, high gain antennas, 60-GHz frequency band.

\section{INTRODUCTION}

$\mathbf{R}$ ECENTLY, the $60-\mathrm{GHz}$ frequency band has received increased interest and attention in the research community due to its potential functional benefits for many upcoming applications. In fact, the development of some new technologies at this frequency band has led to many highspeed wireless developments including high definition video streaming, high-speed internet, high definition multimedia interface, wireless gigabit Ethernet and automotive radars [1], [2]. However, the deployment of millimeter wave technology in this band is affected by the strong oxygen absorption and the high propagation path loss. Thus, one of the key elements for such applications is the antenna, which should be able to

D. Zarifi is with the School of Electrical Engineering, University of Kashan, Kashan, Iran. A. Farahbakhsh is with the School of Electrical Engineering, Graduate University of Advanced Technology, Kerman, Iran. A. U. Zaman and P. S. Kildal are with the Department of Signals and Systems, Chalmers University of Technology, SE-412 96 Göteborg, Sweden (e-mail: zarifi@iust.ac.ir; a_farahbakhsh@iust.ac.ir; zaman@chalmers.se; persimon.kildal@chalmers.se). provide high gain, high radiation efficiency and wide bandwidth characteristics. These specifications imply that both high directivity and fast beam steering is a requirement for some applications. According to [3], for the $60-\mathrm{GHz}$ band, antenna arrays with a gain higher than $30 \mathrm{dBi}$ would be used when the communication distance is larger than $100 \mathrm{~m}$. Another important issue for the system cost reduction is the antenna robustness and ease of integration with other components in a wireless system.

A survey of literature on the millimeter wave antennas indicates that the design of different high-gain antennas for millimeter wave applications has been a subject of extensive research. Different kind of wideband antennas have been designed such as microstrip antennas, parabolic antennas, substrate integrated waveguide (SIW) based planar arrays and slot array antennas. Microstrip arrays are compact, light, easy to manufacture and can easily be integrated with other passive and active components of the wireless system. However, these antennas suffer from high ohmic and dielectric losses and spurious radiation and leakage in the form of surface waves at high frequencies [4-7]. The parabolic antennas have excellent radiation characteristics but because they consist of one or two reflectors, a feed and several supporting feed rods, they are larger and could even be heavier than planar arrays [8]. Besides, the parabolic antenna needs additional fixtures to assemble the transceiver. Recently, some SIW array antennas are developed [9-14]. The antennas based on SIW technology have relatively high radiation efficiency in small arrays and can easily be integrated with other active circuits. Although the losses in SIW are lower than in microstrip structures, the losses is still a great problem, especially for high gain antennas when the SIW array dimension become larger [15].

The waveguide slot antenna arrays are the most attractive candidates for the high-gain wideband planar antennas for many applications [16-20]. They suffer neither from dielectric nor radiation loss and are suitable for applications requiring high gain and high efficiency. However, wideband waveguide slot arrays require corporate feed networks that become very complex and bulky. In addition, at high frequencies, such feed networks require accurate, high precision and expensive manufacturing [21]. In particular it is difficult to provide good electrical contact between different metal layers of the construction. Apart from the problem of metal contact, the antennas need very thin slot layer $(100-200 \mu \mathrm{m})$ which raises mechanical challenges during assembly due to bending of these very thin slot layers. The proposed waveguide slot 


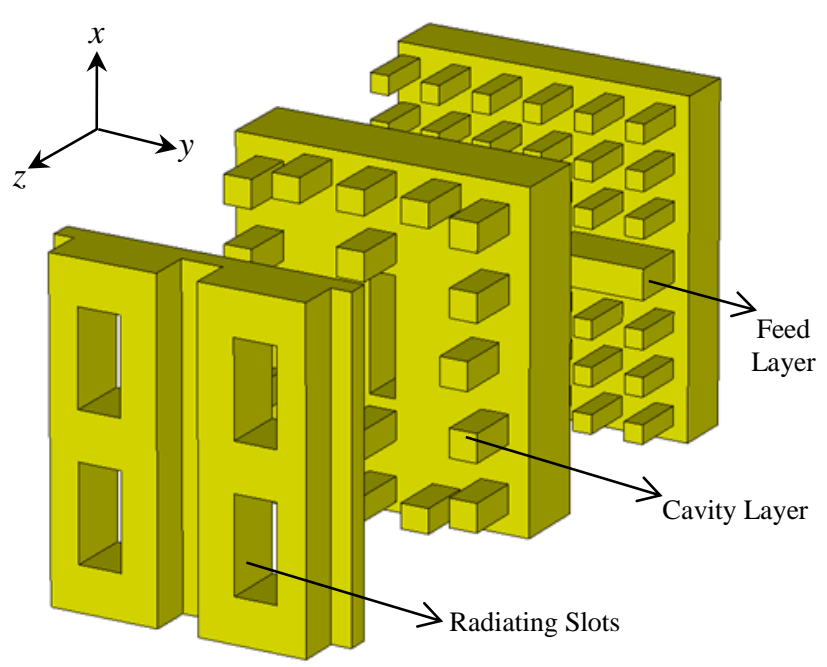

Fig. 1. Exploded 2×2-element sub-array.

antenna arrays in [22-24] have good performances, and they can be manufactured by a promising new their process based on the diffusion bonding of many laminated thin copper plates. Thereby the electrical contact problem between few metal layers is removed, at the expense of having many more very thin metal layers. But these antennas still use very thin slot layers. The present paper solves the electrical contact problem by another promising method, gap waveguides and we propose a new design where the upper slot layer is much thicker and thus mechanically more stable.

The aim of the present work is to design a planar slot antenna array with high-gain and high radiation efficiency based on gap waveguide technology. Our design goal is to cover the bandwidth of $57-66 \mathrm{GHz}$. The gap waveguide technology introduced in [25-28] overcomes the problem of good electrical contact associated with mechanical assembly, by making use of a texture in one of surfaces that creates a magnetic wall (parallel-plate cut-off). There are many modern manufacturing technologies that can be used to produce planar surfaces with small texture, such as die sink Electrical Discharge Manufacturing (EDM), Electron Beam Melting (EBM), multilayer die pressing, and 3D screen-printing. Therefore, the low-cost manufacturing of gap waveguide antennas is possible at high frequencies and therefore, these antennas have large potential for millimeter wave applications.

In fact, the gap waveguide technology uses the cut-off of a PEC-PMC parallel plate waveguide to control desired electromagnetic propagation between the two parallel plates without the requirement of electrical contact. To date, some array antennas have been realized based on gap waveguide technology in 10-15 GHz frequency band [29-31]. The first millimeter wave array antenna was the PCB-based inverted microstrip ridge gap waveguides [32], but it had considerable loss and it was expensive to manufacture all the via holes. In addition, recently, a gap waveguide distribution networks was used in W-band $8 \times 8$ slot arrays $[33,34]$. Here, we present a $16 \times 16$-element slot antenna array with a distribution network based on ridge gap waveguide (RGW) technology.

Organization of this paper is as follows. Section II is devoted to the design of wideband $2 \times 2$-element sub-array. Design procedure of RGW power divider and transitions are discussed in Section III. Section IV deals with the design of complete 16×16-element array, and in Section V details and results of fabrication and measurements are presented. Finally, summary and conclusions are provided in Section VI.

TABLE I. Design PARAMETERS OF 2×2-Element Sub-ARRAY

\begin{tabular}{ccc}
\hline \hline Component & Parameter & Value(mm) \\
\hline \multirow{3}{*}{ Radiating Slot } & length & 2.55 \\
& width & 1.15 \\
& spacing along $x$ & 4.2 \\
& spacing along $y$ & 3.85 \\
Corrugations & width & 1.1 \\
& depth & 1.15 \\
Top Metal Plate & thickness & 1.75 \\
& length & 8.8 \\
& width & 8 \\
Coupling Slot & length & 3.1 \\
& width & 0.7 \\
Ridge & width & 0.8 \\
& height & 1.5 \\
\hline \hline
\end{tabular}

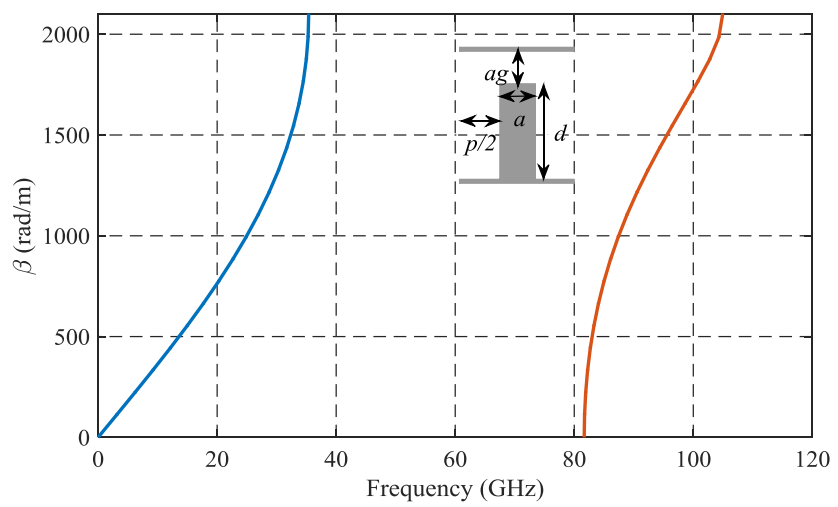

(a)

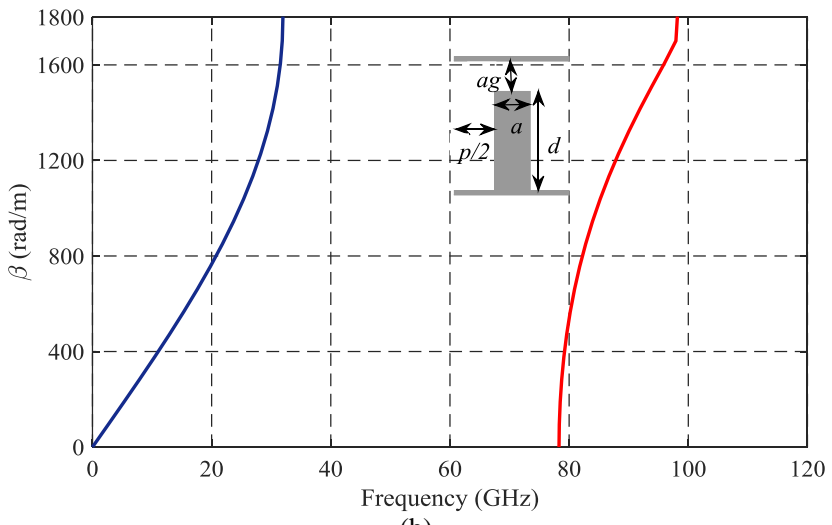

(b)

Fig. 2. Dispersion diagram of the unit cell of the periodic pins used in (a) feed layer $(a=0.5 \mathrm{~mm}, d=1.5 \mathrm{~mm}, p=1 \mathrm{~mm}$ and ag $=0.25 \mathrm{~mm})$ and $(\mathrm{b})$ in cavity layer $(a=0.75 \mathrm{~mm}, d=1.5 \mathrm{~mm}, p=1 \mathrm{~mm}$ and ag $=0.25 \mathrm{~mm})$.

\section{WIDEBAND BASIC $2 \times 2$-ELEMENT SUB-ARRAY}

Fig. 1 shows the configuration of $2 \times 2$-element sub-array which is composed of three parts: the feeding part in the lower layer, the coupling slot and cavity in the middle layer, and the radiating slots in the upper layer. The feeding part is based on 


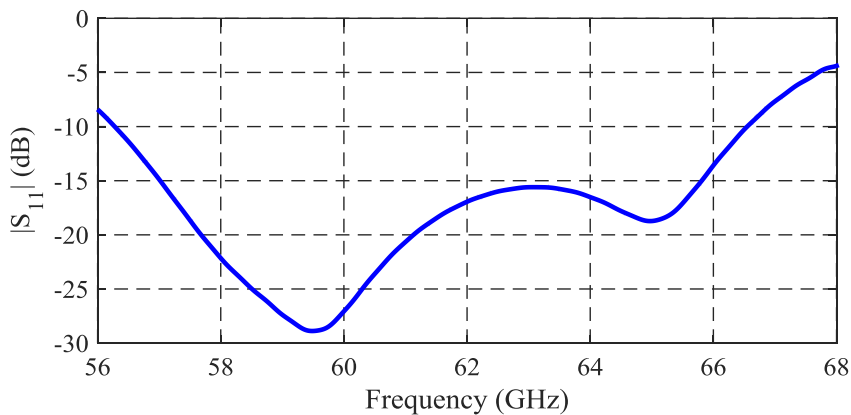

Fig. 3. Simulated reflection coefficient of proposed $2 \times 2$-element sub-array

RGW and can be expanded to a bigger corporate feed network with power dividers and $\mathrm{T}$-junctions. In the next layer, a coupling slot is designed in the metal plane to excite the cavity. In the radiating part, radiating slots are placed as the elements both in the $x$ and $y$ directions on a corrugated metal plane. By proper design of cavity, these four slots are excited equally in amplitude and phase to give a broadside beam. In Table I, the design parameters of $2 \times 2$-element sub-array are listed.

Notice that from the practical point of view, using a thin metal plate in the radiating layer usually led to some problems in fabrication and assembling process which may affect on the antenna performance. On the other hand, a thick layer deteriorates reflection coefficient of the antenna. To overcome this problem and also decreasing grating lobes, some corrugations are created in the radiating layer. The width and depth of these corrugations are optimized to improve both the $\mathrm{S}_{11}$ and the radiation pattern of the antenna. The corrugations work as a soft surface, thereby stopping waves from coupling into the neighboring parallel slots [35].

The pin dimensions in the feed and cavity layers are chosen according to the guidelines in [27] to be $0.5 \times 0.5 \times 1.5 \mathrm{~mm}^{3}$ and $0.75 \times 0.75 \times 1.5 \mathrm{~mm}^{3}$, respectively. The period of pins and the air gap between the pins and upper metal plate are chosen to be 1 and $0.25 \mathrm{~mm}$, respectively, to achieve a stop-band approximately from 40 to $80 \mathrm{GHz}$. The dispersion diagrams of the infinite two-dimensional pin array are shown in Fig. 2.

Full-wave simulation of the designed subarray is performed by using the time-domain solver of the CST Microwave Studio which uses a finite element method. In order to consider the mutual coupling in the infinite two-dimensional slot array, the periodic boundary conditions in both $x$ and $y$ directions are placed. The simulated reflection coefficient is shown in Fig. 3. The results show about $17 \%$ of reflection coefficient bandwidth $\left(\left|\mathrm{S}_{11}\right|<-10 \mathrm{~dB}\right)$ in the frequency band of 56.2-66.6 GHz.

\section{POWER DIVIDER AND TRANSITION DESIGN}

\section{A. Ridge Gap Waveguide Power Divider}

In order to expand the designed sub-array to a bigger structure, we need to a corporate feed network including power dividers and $\mathrm{T}$-junctions. The configuration of a fourway RGW power divider is illustrated in Fig. 4(a). Observe that due to the symmetry and using $90^{\circ}$ bends in this structure, the output ports have constant amplitude and phase. The

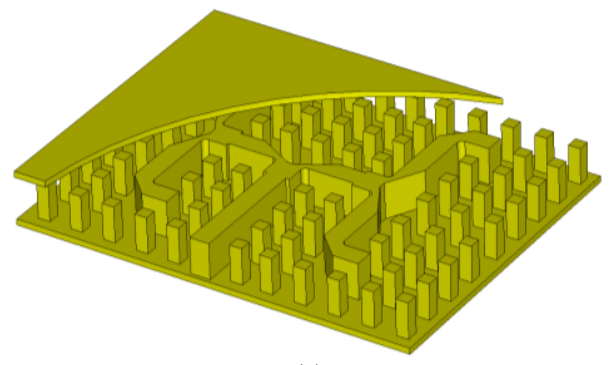

(a)

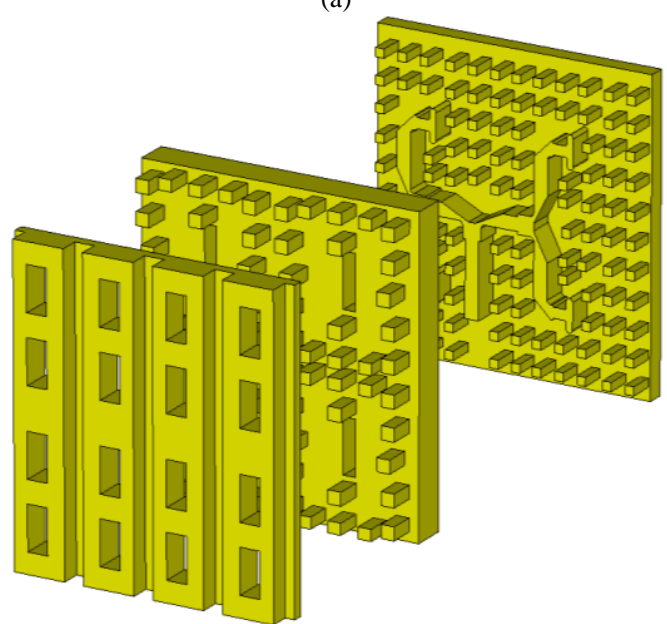

(b)

Fig. 4. Schematic of the (a) 4-way power divider based on RGW, and (b) $4 \times 4$-elementa array antenna. The length and width of impedance matching sections in T-junctions are chosen 0.925 and $1.1 \mathrm{~mm}$. Also, the lengths of bases in V-shape junctions are 0.4 and $3.25 \mathrm{~mm}$. The distances between the output ports along the $\mathrm{x}$ - and $\mathrm{y}$-axis are 8 and $8.8 \mathrm{~mm}$, respectively.

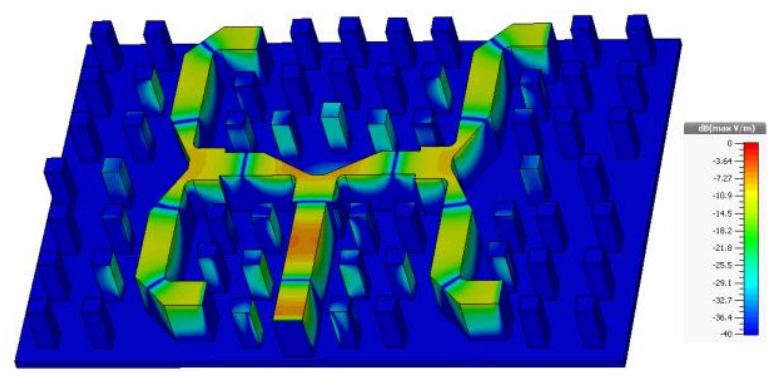

Fig. 5. Electric field distribution of 4-way power divider at $60 \mathrm{GHz}$.

electric field distribution at frequency $60 \mathrm{GHz}$ is presented in Fig. 5. The simulated reflection coefficient of this structure is depicted in Fig. 6. Observe that there is a very good $S_{11}$ and a very small amplitude imbalance (in the worst case $-0.25 \mathrm{~dB}$ ) between the output ports of power divider. Thus, this power divider can be expanded to design a bigger corporate feeding network based on RGW technology.

Using the proposed 4-way power divider, we can design a $4 \times 4$-element array antenna as shown in Fig. 4(b). Notice that in order to achieve better coupling between the RGW and the coupling slot and to improve the $S_{11}$ of the structure, some Tshaped impedance matching sections are added to the output ports of the power divider. Fig. 6 shows the simulated reflection coefficient of this antenna. The results indicate about $17 \%$ impedance bandwidth $(56.5-67 \mathrm{GHz})$ for $\mathrm{SWR} \leq 2$. 


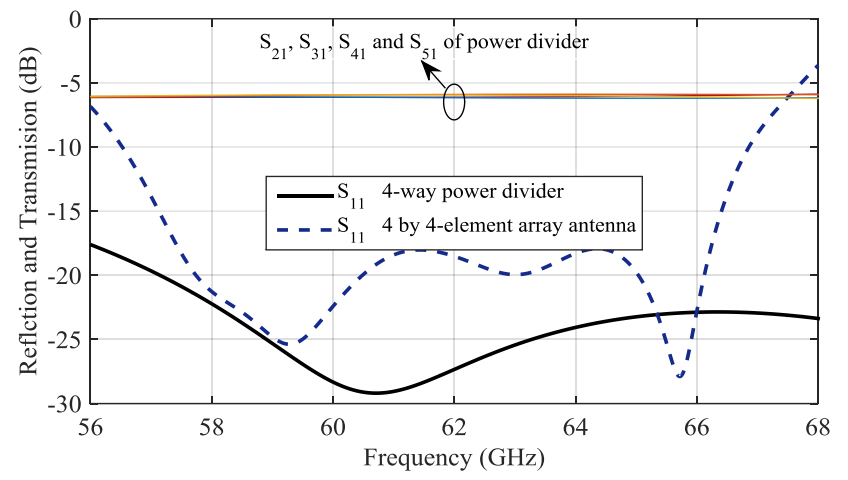

Fig. 6. Simulated reflection and transmission coefficients of 4-way power divider and the reflection coefficient of $4 \times 4$-element array antenna.
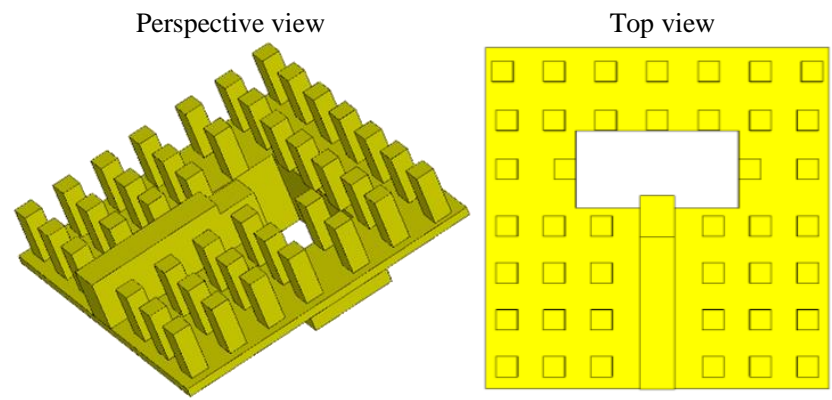

(a)

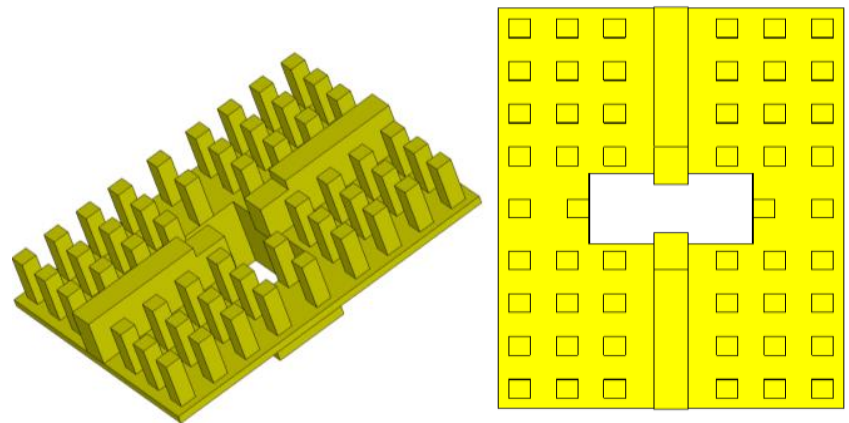

(b)

Fig. 7. RWG to WR-15 transition. (a) Non-symmetric transition, and (b) symmetric transition.The length and height of step in non-symmetric (symmetric) transitions are $1 \mathrm{~mm}(1.37 \mathrm{~mm})$ and $0.32 \mathrm{~mm}(0.15 \mathrm{~mm})$.

\section{B. Transition from $R G W$ to $W R-15$}

The antenna is excited with a standard V-band rectangular waveguide (e.g. WR-15) from the bottom plane. The transition transforms the electromagnetic fields from the rectangular waveguide mode to the RGW mode. Here, we propose two structures, one being geometrically symmetric and the other asymmetric. The overall geometries of these transitions are shown in Fig. 7. It is clear that the symmetrical transition has two output ports that have $180^{\circ}$ phase difference. Observe that we make a matching step at the end of each ridge to achieve a good matching between RGW to WR-15. By fine tuning the location of the pins around the WR-15 opening, and the height and length of the stub, we are able to match the RGW to WR15 over the desired bandwidth of 57-66 GHz. The simulated reflection coefficient and insertion loss for these two transitions are shown in Fig. 8. The results show about $17 \%$ reflection coefficient bandwidth $\left(\left|S_{11}\right|<-20 \mathrm{~dB}\right)$ in the frequency band of 56-66 GHz.

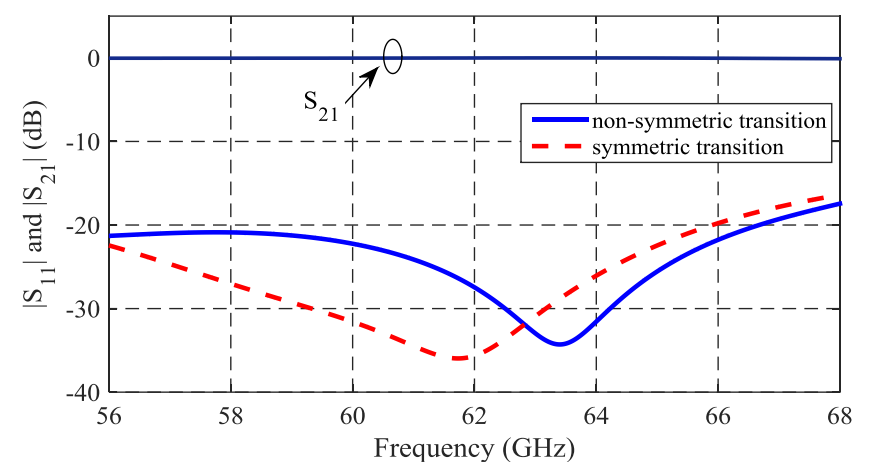

Fig. 8. Reflection coefficient of non-symmetric and symmetric transition from RGW to WR-15.

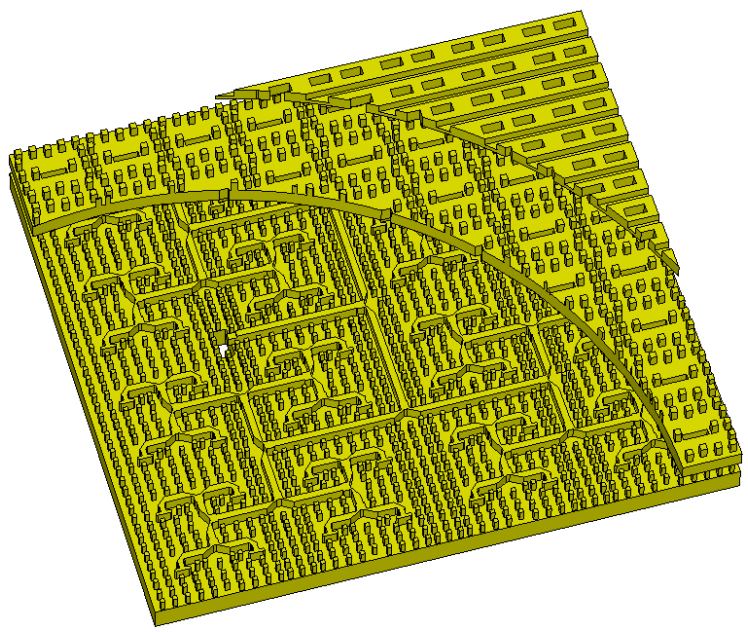

(a)

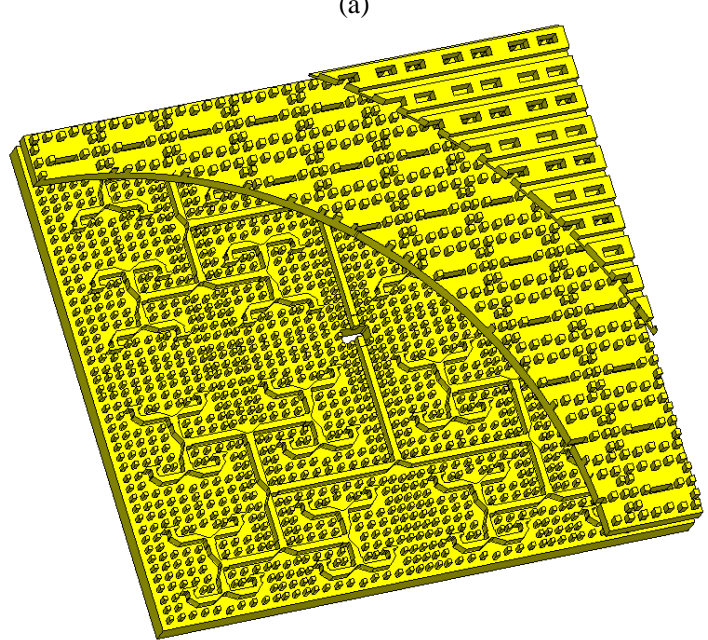

(b)

Fig. 9. Configuration of $16 \times 16$-element array antenna with (a) nonsymmetric transition, and (b) symmetric transition from RGW to WR15.

\section{DESIGN OF COMPLETE $16 \times 16$-ELEMENT ARRAY}

Fig. 9 shows the geometric configuration of the proposed $16 \times 16$-element slot array antenna. A 64-way RGW power divider is deployed to feed 64 cavities, each of which excites four radiating slots. The overall corporate distribution network is analyzed and optimized to compensate for the mutual coupling from adjacent discontinuities in the radiating layer. As shown in Fig. 9(b), when we use the symmetric Ttransition in the structure, the structure must be symmetric 
TABLE II. DESIGN PARAMETERS OF 16×16-ELEMENT ARRAY ANTENNA

\begin{tabular}{ccc}
\hline \hline Component & Parameter & Value(mm) \\
\hline \multirow{2}{*}{ Radiating Slot } & length & 2.6 \\
& width & 1.35 \\
& round radius & 0.2 \\
Corrugations & width & 1.1 \\
& depth & 1.15 \\
Top Metal Plate & thickness & 1.75 \\
& length & 80 \\
& width & 80 \\
Coupling Slot & length & 3.15 \\
& width & 0.7 \\
Middle Metal Plate & round radius & 0.2 \\
& thickness & 2 \\
Transition & length of step & 1.37 \\
& height of step & 0.15 \\
Bottom Metal Plate & thickness & 5 \\
& & \\
Feed Waveguide & length & 3.8 \\
& width & 1.9 \\
\hline \hline
\end{tabular}

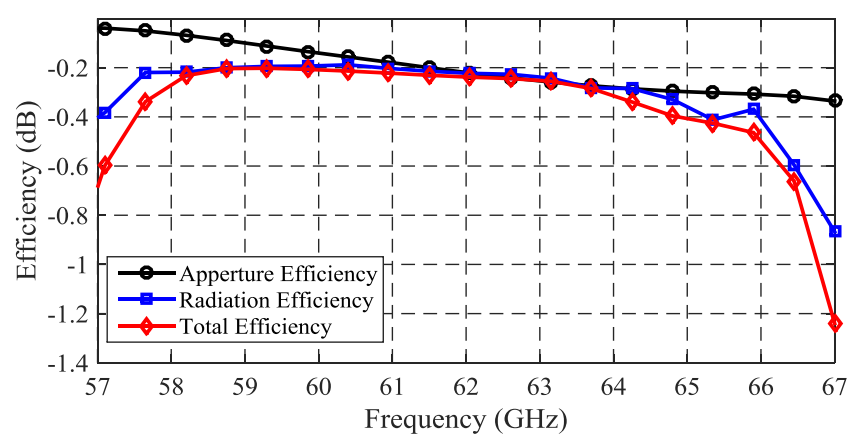

Fig. 10. Computed aperture efficiency, radiation efficiency and total radiation efficiency of the $16 \times 16$-element array antenna.

along the $x$-axis in order to compensate for the $180^{\circ}$ phase difference of the waves leaving the transition,. This configuration has some advantages: from the practical point of view, the WR-15 port is placed in the center. From the design point of view, a T-junction is removed from the structure which results in improving $S_{11}$. And, finally, because of the symmetry, only a quarter of the antenna needs to be meshed for the full-wave simulations in CST. The latter leads to much shorter computation time and memory consumption.

The aperture efficiency, radiation efficiency and total radiation efficiency of the proposed array antenna are computed and illustrated in Fig. 10. The simulated reflection coefficient and radiation pattern of the complete antenna along with the experimental results will be presented in the next section.

\section{ANTENNA FABRICATION AND EXPERIMENTAL RESULTS}

\section{A. Fabrication of $16 \times 16$-element Array}

To verify the performance of the designed array antenna, a prototype of the $16 \times 16$-element array antenna with dimension of $80 \mathrm{~mm} \times 80 \mathrm{~mm}$ (dimensions of aperture are $70.4 \times 64$ $\mathrm{mm}^{2}$ ) was fabricated in aluminum by milling. The complete
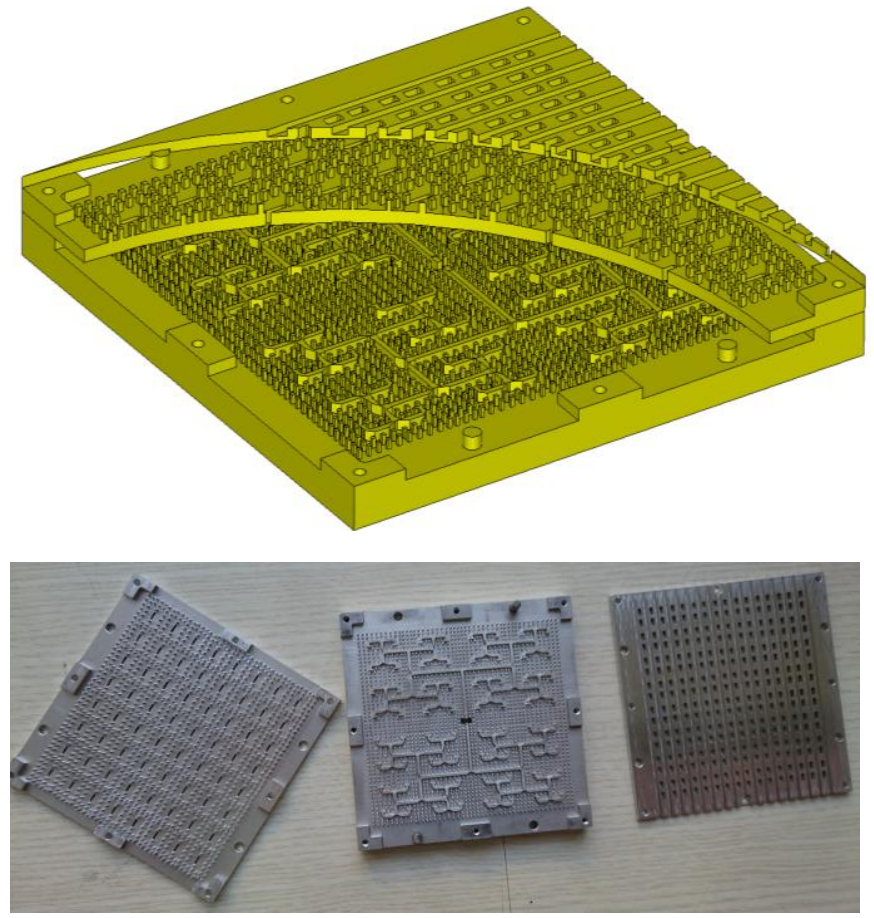

Fig. 11. Final configuration of $16 \times 16$-element array and photograph of fabricated antenna. The structure has dimension of $80 \times 80 \times 12.2 \mathrm{~mm}^{3}$.
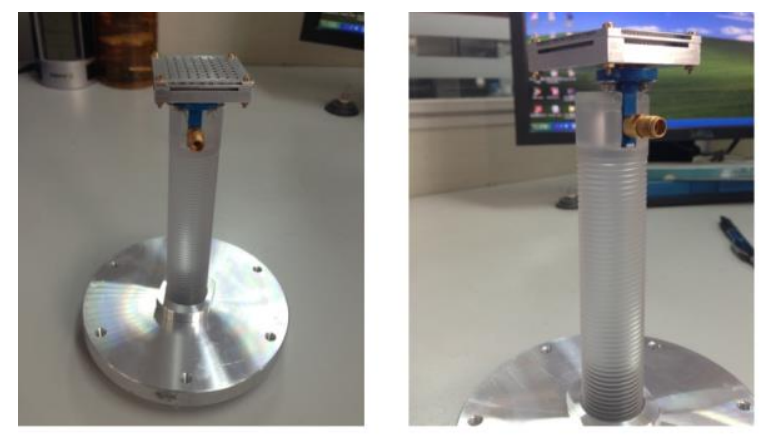

Fig. 12. Photograph of the under test antenna in the measurement setup.

antenna configuration including holes for screws and alignment pins are presented in Fig. 11, together with photos of the three manufactured parts. Notice that due to the limitations in the fabrication process, all sharp corners and edges have been rounded with minimum radius $0.2 \mathrm{~mm}$. Thus, according to this matter, the antenna structure including feed layer, cavity layer and radiating layer, is optimized again, and the final antenna design parameters are listed in Table II. Also, notice that the $5.0 \mathrm{~mm}$ thickness of the bottom metal plate is not required for the antenna operation and it was chosen only to be able to connect to a standard WR-15 flange with screws.

\section{B. Array Antenna Performance}

The fabricated array antenna reflection coefficient was measured using a Vector Network Analyzer connected to the WR-15 input port. The antenna is shown in Fig. 12. The measured and simulated input reflection coefficients are shown in Fig. 13. Observe that the fabricated antenna has reflection coefficient below $-10 \mathrm{~dB}$ over the frequency band of 56-65.7 GHz. There are some differences between the 


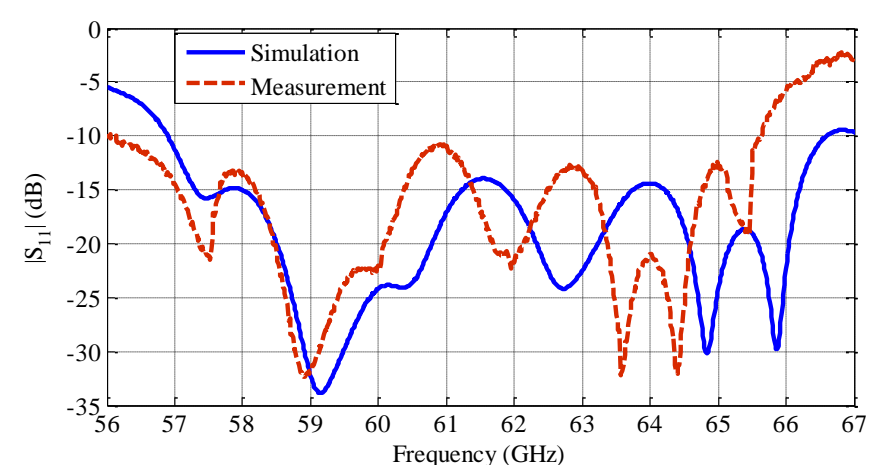

Fig. 13. Simulated and measured reflection coefficient and gain of fabricated 16×16-element array antenna.

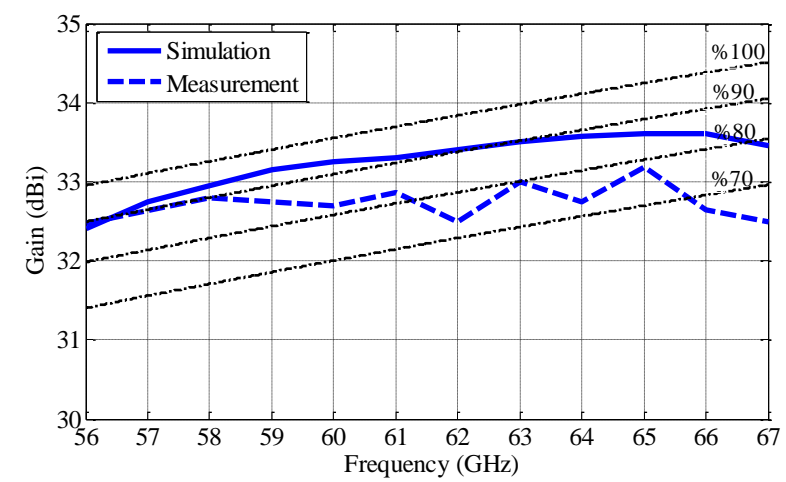

Fig. 14. Simulated and measured reflection coefficient and gain of fabricated 16×16-element array antenna.

simulated and measured results, but generally they are in quite good agreement. The differences are mainly due to manufacturing and assembling tolerances.

The radiation patterns and gains of the array antenna are measured in an outdoor test range with the use of a V-band antenna measurement system. The simulated and measured frequency characteristics of the realized boresight gain are illustrated in Fig. 14. The dotted lines show that the maximum available directivity of an aperture having dimension of $70.4 \times$ $64 \mathrm{~mm}^{2}$ and when aperture efficiency is 100, 90, 80 and $70 \%$. The obtained gain is more than $32.5 \mathrm{dBi}$ with a measured efficiency higher than $70 \%$. The gain response is quite flat, with a gain variation of $0.7 \mathrm{~dB}$ over the $56-66 \mathrm{GHz}$ bandwidth. The difference between the measured and simulated gain results may be owing to extra reflection loss and extra ohmic losses due to metal conductivity degradation with surface roughness.

The simulated and measured radiation patterns of antenna at 58,62 and $66 \mathrm{GHz}$ in both the E- and H-planes and also $45^{\circ}$ plane are shown in Figs. 15-17. We see that the measured patterns show excellent agreement with the simulated ones. The simulated and measured radiation patterns are symmetrical and the first sidelobe levels in both $\mathrm{E}-$ and $\mathrm{H}$ planes are around $-13 \mathrm{~dB}$. The measured side lobe levels of the fabricated array in the $45^{\circ}$-plane are below $-25 \mathrm{~dB}$ over the desired frequency band.

\section{SUMMARY AND CONCLUSION}

In summary, we have designed and studied numerically and experimentally a wideband high gain high efficiency slot

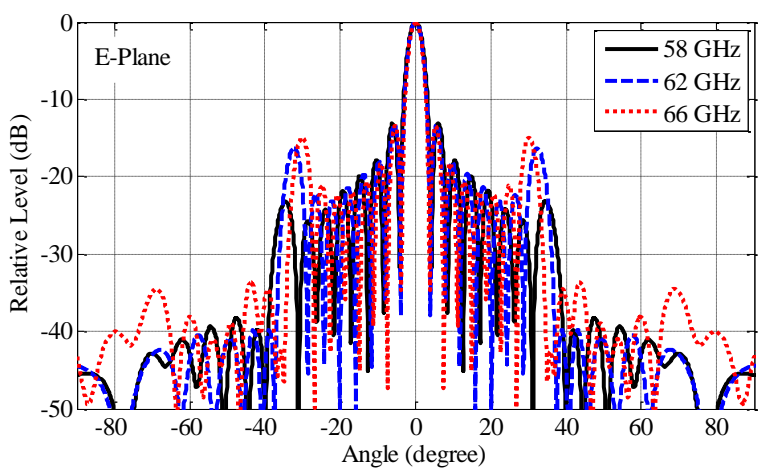

(a)

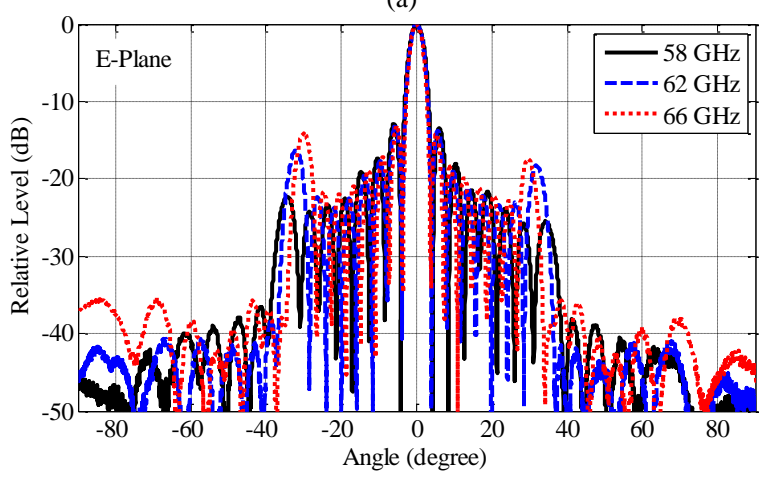

(b)

Fig. 15. Radiation patterns of the fabricated array at E-plane and frequencies 58, 62 and $66 \mathrm{GHz}$. (a) Simulated, (b) measured.

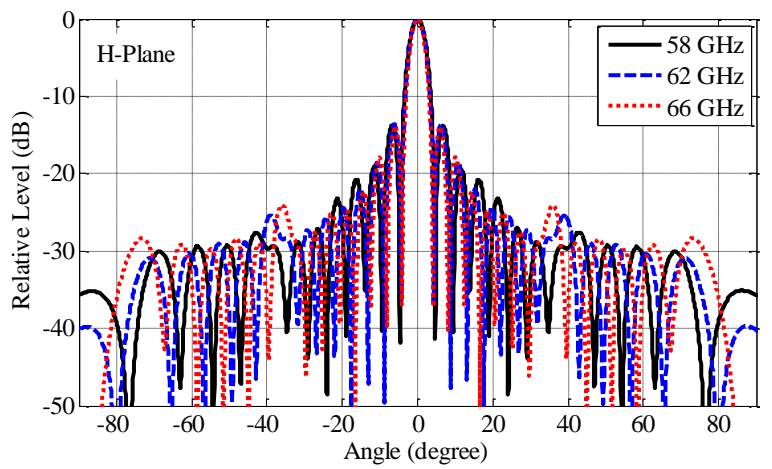

(a)

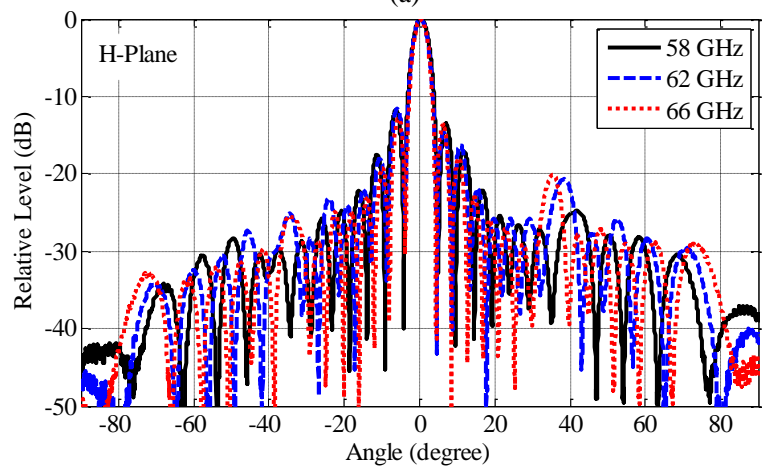

(b)

Fig. 16. Radiation patterns of the fabricated array at H-plane and frequencies 58, 62 and $66 \mathrm{GHz}$. (a) Simulated, (b) measured.

antenna array for V-band. In this work, we used a new corporate feed network based on the ridge gap waveguide technology. The gap waveguide technology eliminates the requirement of electrical contact between the different layers 


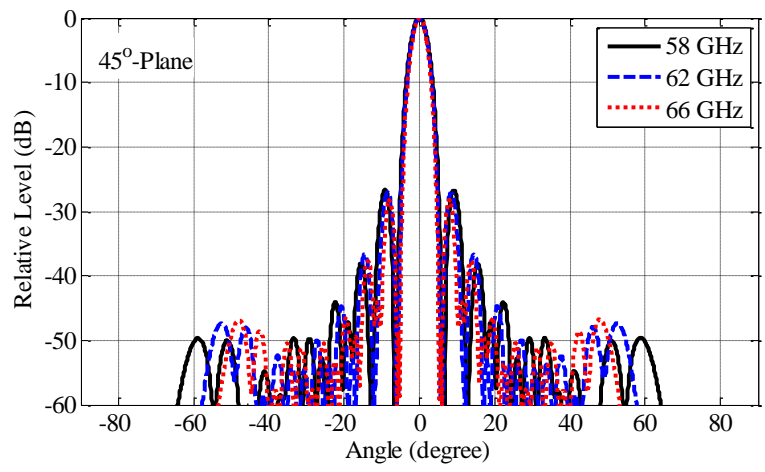

(a)

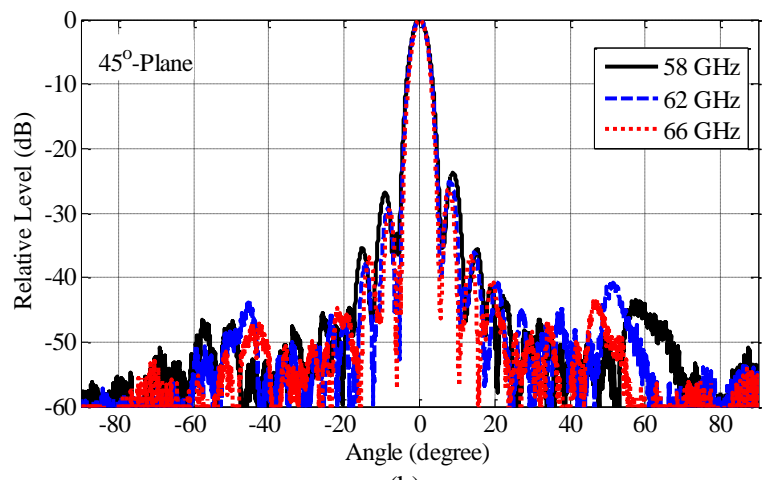

(b)

Fig. 17. Radiation patterns of the fabricated array at $45^{\circ}$-plane and frequencies 58, 62 and 66 GHz. (a) Simulated, (b) measured.

of a multilayer antenna structure. Two transition structures between ridge gap waveguide and WR-15 waveguide have been designed in order to provide a simple excitation of the antenna. In addition, we propose a new design where the upper slot layer is much thicker and thus mechanically more stable. Finally, a $16 \times 16$-element array was designed, simulated, fabricated and measured. The measured realized gain is higher than $32.5 \mathrm{dBi}$ over the entire operation bandwidth from 56 to $65.7 \mathrm{GHz}$, corresponding to aperture efficiency larger than $70 \%$.

\section{ACKNOWLEDGMENT}

The work has been supported by the European Research Council (ERC) via an advanced investigator grant ERC-2012ADG 20120216, and by a project within the VINNOVA funded Chase Antenna Systems excellence center at Chalmers.

\section{REFERENCES}

[1] P. Smulders, "Exploiting the $60 \mathrm{GHz}$ band for local wireless multimedia access: Prospects and future directions," IEEE Commun. Mag.vol. 40, no. 1, pp. 140-147, Jan. 2002.

[2] S. K. Yong and C.-C. Chong, "An overview of multigigabit wireless through millimeter wave technology: Potentials and technical challenges," EURASIP J. Wire. Comm. Netw., vol. 2007, pp. 1-10, 2007.

[3] Y. P. Zhang and D. X. Liu, "Antenna-on-chip and antenna-in-package solutions to highly integrated millimeter-wave devices for wireless communications," IEEE Trans. Antennas Propag., vol. 57, no. 10, pp. 2830-2841, Oct. 2009.

[4] E. Levine, G. Malamud, S. Shtrikman, and D. Treves, "A study of microstrip array antenna with the feed network," IEEE Trans. Antennas Propag., vol. 37, no. 4, pp. 426-434, Apr. 1989.

[5] M. Li, K. M. Luk, "Low-cost wideband microstrip antenna array for 60GHz applications,” IEEE Trans. Antennas Propag., vol. 62, no. 6,pp. 3012-3018, June 2014.
[6] A. Lamminen, J. Saily, and A. R. Vimpari, "60 GHz patch antennas and arrays on LTCC with embedded cavity substrates," IEEE Trans. Antennas Progag., vol. 56, no. 9, pp. 2865-2874, Sep. 2008.

[7] A. Bondarik, D. S. Jun, J. M. Kim, "investigation of microstrip antenna array stacked structure realized on LTCC for $60 \mathrm{GHz}$ band," Microw. Opt. Tech. Lett., vol. 52, no. 3,pp. 648-652, 2010.

[8] M.Nagasaka, S.Nakazawa, andS .Tanaka,"12/21GHzdual-bandfeed antenna for satellite broadcasting receiving reflector antenna," inProc.ISAP, 2012, pp. 790-793.

[9] X. P. Chen, K. Wu, L. Han, and F. H. , "Low-cost high gain planar antenna array for $60-\mathrm{GHz}$ band applications," IEEE Trans. Antennas Propag., vol. 58, no. 6, pp. 2126-2129, Jun. 2010.

[10] M. Bozzi, A. Georgiadis, and K. Wu, "Review of substrate-integrated waveguide circuits and antennas," IET Microw., Antennas Propag., vol. 5, no. 8, pp. 909-920, Jun. 2011.

[11] S. Cheng, H.Yousef, and H. Kratz, "79 GHz slot antennas based on substrate integrated waveguides (SIW) in a flexible printed circuit board,"IEEE Trans. Antennas Propag., vol. 57, no. 1, pp. 64-71, 2009.

[12] J. F. Xu, Z. N. Chen, X. M. Qing, and W. Hong, "Bandwidth enhancement for a $60 \mathrm{GHz}$ substrate integrated waveguide fed cavity array antenna on LTCC," IEEE Trans. Antennas Progag., vol. 59, no. 3, pp. 826-832, Mar.2011.

[13] J. Wu, Y. J. Cheng, Y. Fan, "A wideband high gain high efficiency hybrid integrated plate array antenna for V-band inter satellite links," IEEE Trans. Antennas Propag., vol. 63, no. 4,pp. 1225-1233, April 2015.

[14] J. Wu, Y. J. Cheng, Y. Fan, “60-GHz substrate integrated waveguide fed cavity-backed aperture-coupled microstrip patch antenna array," IEEE Trans. Antennas Propag., vol. 63, no. 3,pp. 1075-1085, March 2015.

[15] M. Bozzi, M. Pasian, L. Perregrini, and K.Wu, "On the losses in substrate integrated waveguides and cavities," Int. J. Microw. Wireless Tech., vol. 5, no. 1, pp. 395-401, 2009.

[16] S. Park, Y. Tsunemitsu, J. Hirokawa, and M. Ando, "Center feed single layer slotted waveguide array," IEEE Trans. Antennas Propag., vol. 54, no. 5, pp. 1474-1480, May 2006.

[17] J. Hirokawa and M. Ando, "Efficiency of 76-GHz post-wall waveguide fed parallel-plate slot arrays, 'IEEE Trans. Antennas Propag. vol. 48, no. 11, pp. 1742-1745, Nov. 2000.

[18] S. Fujii, Y. Tsunemitsu, G. Yoshida, N. Goto, M. Zhang, J. Hirokawa, and M. Ando, "A wideband single-layer slotted waveguide array with an embedded partially corporate feed," in Proc. Int. Symp. Antennas Propag., TP-C27-5, Oct. 2008.

[19] T. Tsugawa, Y. Sugio, and Y. Yamada, "Circularly polarized dielectricloaded planar antenna excited by the parallel feeding waveguide network," IEEE Trans. Broadcasting, vol. 43, no. 2, pp. 205-212, Jun. 1997.

[20] G. L. Huang, S. G. Zhou, T. H. Chio, H. T. Hui, and T. S. Yeo, "A Low Pro file and Low Sidelobe Wideband Slot Antenna Array Feb by an Amplitude-Tapering Waveguide Feed-Network," IEEE Trans. AntennasProgag., vol. 63, no. 1, pp. 419-423, Jan. 2015.

[21] Y. Kimura, T. Hirano, J. Hirokawa, and M. Ando, "Alternating-phase fed single-layer slotted waveguide arrays with chokes dispensing with narrow wall contacts," in IEE Proc.-Microw. Antennas Propag, Oct. 2001, vol. 148, no. 5.

[22] Y. Miura, J. Hirokawa, M. Ando, Y. Shibuya, and G. Yoshida, "Doublelayer full-corporate-feed hollow-waveguide slot array antenna in the 60 GHz-band," IEEE Trans. Antennas Propag., vol. 59, no. 8, pp. 28442851, Aug. 2011.

[23] K. Dongjin, J. Hirokawa, M. Ando , J. Takeuchi, and A. Hirata, "64×64element and $32 \times 32$-element slot array antennas using double-layer hollow-waveguide corporate-feed in the $120 \mathrm{GHz}$ band," IEEE Trans. Antennas Propag. , vol. 62, no. 3, pp. 1507-1512, Mar. 2014.

[24] D. Kim, M. Zhang, J. Hirokawa, andM. Ando, "Design and fabrication of a dual-polarization waveguide slot array antenna with high isolation and high antenna efficiency for the $60 \mathrm{GHz}$ band," IEEE Trans. Antennas Propag. , vol. 62, no. 6, pp. 3019-3027, June 2014.

[25] P.-S. Kildal, E. Alfonso, A. Valero-Nogueira, and E. Rajo-Iglesias, "Local metamaterial-based waveguides in gaps between parallel metal plates," IEEE Antennas Wireless Propag. Lett., vol. 8, pp. 84-87, 2009.

[26] P.-S. Kildal, "Three metamaterial-based gap waveguides between parallel metal plates for $\mathrm{mm} / \mathrm{submm}$ waves," in Proc. 3rd Eur. Conf. Antennas Propag., Berlin, Mar. 2009.

[27] E. Rajo-Iglesias and P.-S. Kildal, "Numerical studies of bandwidth of parallel plate cut-off realized by bed of nails, corrugations and 
mushroom-type EBG for use in gap waveguides," IET Microw., Antennas Propag., vol. 5, no. 3, pp. 282-289, Mar. 2011.

[28] P.-S. Kildal, A. U. Zaman, E. Rajo-Iglesias, E. Alfonso, and A. ValeroNogueira, "Design and experimental verification of ridge gap waveguides in bed of nails for parallel plate mode suppression," IETMicrow., Antennas Propag., vol. 5, no. 3, pp. 262-270, Mar. 2011.

[29] A. U. Zaman and P.-S. Kildal, "Slot antenna in ridge gap waveguide technology," in Proc. 6th Eur. Conf. Antennas Propag., Prague, Czech Republic, Mar. 2012.

[30] A. U. Zaman and P. S. Kildal, "Wide-Band Slot Antenna Arrays With Single-Layer Corporate-Feed Network in Ridge Gap Waveguide Technology," IEEE Trans. Antennas Propag., vol. 62, no. 6, pp. 29923001, June 2014

[31] E. Pucci, E. Rajo-Iglesias, J. L. Vazquez-Roy, and P. S. Kildal, "Planar Dual-Mode Horn Array With Corporate-Feed Network in Inverted Microstrip Gap Waveguide," IEEE Trans. Antennas Propag. , vol. 62, no. 6, pp. 3534-3542, 2014.
[32] A. Razavi, P.-S. Kildal, X. Liangliang, E. Alfonso, and H. Chen, "2x2slot Element for $60 \mathrm{GHz}$ Planar Array Antenna Realized on Two Doubled-sided PCBs Using SIW Cavity and EBG-type Soft Surface fed by Microstrip-Ridge Gap Waveguide," IEEE Trans. Antennas Propag. , vol. 62 , no. 9, pp. 4564-4573, Sep. 2014.

[33] B. Cao, H. Wang, and Y. Huang, "W-band High Gain TE $220-$ Mode Slot Antenna Array with Gap Waveguide Feeding Network," IEEE Trans. Antennas Propag, Early access, 2015.

[34] B. Cao, H. Wang, Y. Huang, and J. Zheng, "High-Gain L-Probe Excited Substrate Integrated Cavity Antenna Array with LTCC-based Gap Waveguide Feeding Network for W-band Application," IEEE Trans. Antennas Propag, Early access, 2015.

[35] P.-S. Kildal, A. A. Kishk, and S. Maci, "Special issue on artificial magnetic conductors, soft/hard surfaces, and other complex surfaces" (Guest Editorial), IEEE Transactions on Antennas and Propagation, vol. 53, no. 1, pp. 2-7, Jan. 2005. 